\title{
Pain in the Neck Following Colonoscopy
}

\section{Vanessa Cubas ${ }^{1 *}$, and Peter William Waterland ${ }^{2}$}

${ }^{1}$ Surgical Core Trainee (CT1 colorectal surgery), Birmingham Heartlands Hospital, United Kingdom

${ }^{2}$ Surgical Registrar (ST7 colorectal surgery), Birmingham Heartlands Hospital, United Kingdom

\begin{abstract}
Colonoscopy remains a widely used diagnostic and therapeutic procedure. However, like any invasive investigation it has the potential of complications; extraperitoneal perforation with pneumoretroperitoneum and subcutaneous emphysema being an extremely rare example.

We report such a case in a57 year old woman who presented to the emergency department with abdominal pain and diffuse chest, neck and facial surgical emphysema following a routine colonoscopy with hot snarepolypectomy.
\end{abstract}

\section{Keywords:}

Colonoscopy; Pain in the neck

\section{Introduction}

About 1 in 20 people in the UK develop bowel cancer during their lifetime. It remains the third most common cancer in the UK and the second leading cause of cancer deaths. Colonoscopy remains the gold standard investigation in the diagnosis of colon cancer and is routinely performed as part of the NHS bowel cancer screening programme.

However, complications such as perforation, bleeding and post-polypectomy syndrome may variably occur.The incidence of colonic perforation ranges from $0.016 \%$ to $0.2 \%$ following diagnostic colonoscopy and may be up to $5 \%$ following some colonoscopic interventions [1] Perforations occur more often during therapeutic colonoscopy and in patients of advanced age or with multiple comorbidities.

Colonic perforation presenting with pneumoretroperitoneum, pneumomediastinum and subcutaneous emphysema is extremely rare [2]. We report the case of a patient presenting with abdominal pain and dysphonia associated with diffuse chest, neck and facial surgical emphysema following a colonoscopy.

\section{Case Presentation}

A 57-year-old woman attended the emergency department complaining ofleft sidedabdominal pain and swelling of the neck, face, and chest associated with minorbreathing difficulties. According to her partner, her voice sounded different in pitch to normal.

Three hours prior to admission she underwent a colonoscopy for investigation of a change in bowel habit. At colonoscopy the endoscopist noted scattered diverticulosis of the left colon and a $10 \mathrm{~mm}$ descending colonic sessile polyp which was excised and retrieved with a hot snare.

On examination a low gradepyrexia was noted and her respiratory rate was slightly elevated (RR 18, oxygen saturation $96 \%$ on room air). Palpable surgical emphysema with crepitus was notedover her face, neck and upper chest, which was tender. Her abdomen was soft with localised tenderness over the left flank and iliac fossa.

Abnormal laboratory findings included a mildly raised white cell count $\left(11.2610^{9} / \mathrm{l}\right)$ and a minimally elevated C-reactive protein $(10 \mathrm{mg} / \mathrm{L})$. The patient underwent chest radiograph and chest and abdominal computed tomography (CT) scan. Right subdiaphragmatic air and diffuse subcutaneous emphysema were observed (Figure 1).
The CT scan showed possible pneumoperitoneum, very significant retropneumoperitoneum, pneumomediastinum with and upper thoracic, neck and facial surgical emphysema likely secondary to a bowel perforation in the proximal descending colon.Incidently a right upper lobe lung lesion suspicious for primary malignancy was also noted with adjacent hilar lymph nodes but no evidence of distant metastases (Figures 2-4).

Initial management involved oxygen therapy, intravenous fluid and antibiotics (Amoxicillin, Gentamycin and Metronidazole).

The next day clinical examination revealed generalised abdominal tenderness with guarding and rebound tenderness.Surgery was decided in view of the spreading abdominal signs and a laparoscopy performed. Interestingly, laparoscopy was surprisingly normal, with

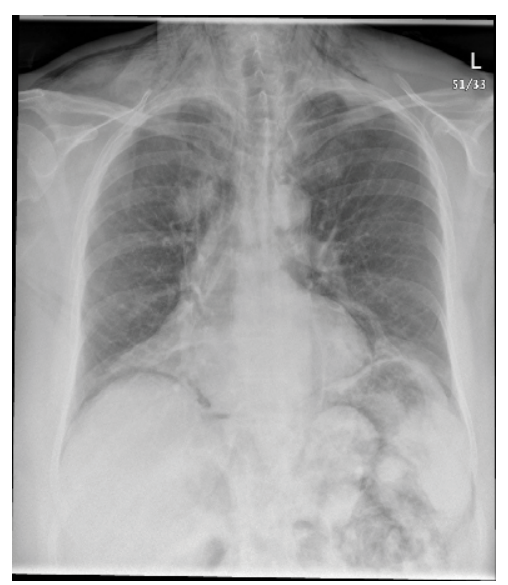

Figure 1: Chest X-ray Demonstrating Diffuse subcutaneous Emphysema and Subdiaphragmatic Retroperitoneal Free Air

*Corresponding author: Vanessa Cubas, Surgical Core Trainee (CT1 colorectal surgery), Birmingham Heartlands Hospital, United Kingdom, Tel: +44 121 7729456671; E-mail: vanessacubas@nhs.net

Received October 13, 2014; Accepted November 24, 2014; Published November 24, 2014

Citation: Cubas V, Waterland PW. Pain in the Neck Following Colonoscopy. Journal of Surgery [Jurnalul de chirurgie] 2014; 10(3): 247-249 DOI: 10.7438/15849341-10-3-11

Copyright: @ 2014 Cubas V, et al. This is an open-access article distributed under the terms of the Creative Commons Attribution License, which permits unrestricted use, distribution, and reproduction in any medium, provided the original author and source are credited. 


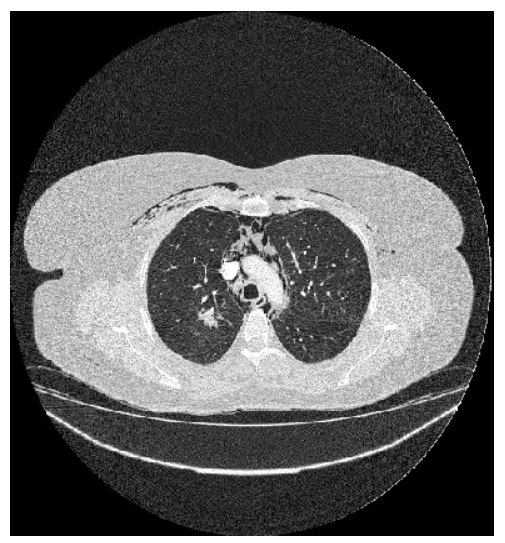

Figure 2: Axial CT Image of the Chest Revealing Bilateral Diffuse Subcutaneous Emphysema and Pneumomediastinum. Free Air is Noted at the Anatomical Region of the Aortic Arch

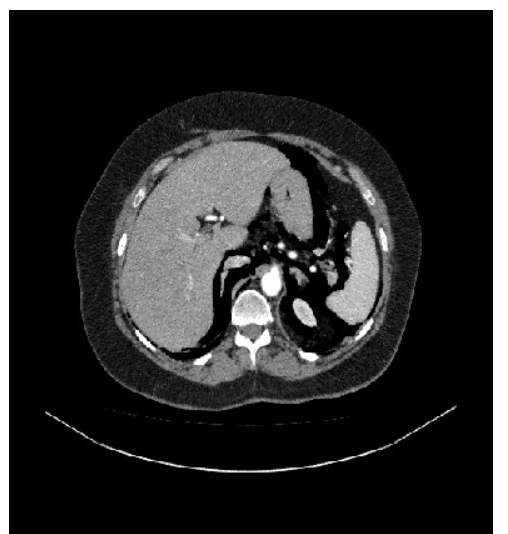

Figure 3: Axial CT Slice of the Upper Abdomen Demonstrating Pneumoperitoneum. Free Air Envelops the Retroperitoneal Structures

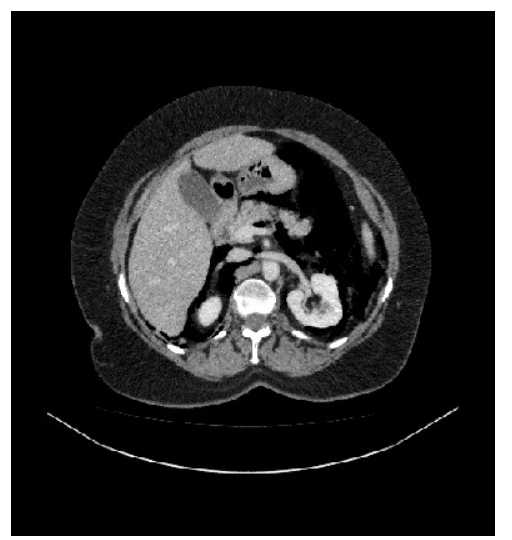

Figure 4: Extensive Amount of Retroperitoneal Air Displacing the Descending Colon Anteriorly

no contamination seen and only minimal amounts of pelvic free fluid identified. A laparoscopic aspiration and lavage was performed and tube drains left in situ as a precaution. The post-operative course was uneventful and she was discharged four days later.

\section{Discussion}

Colonoscopy is a common and usually a very safe diagnostic and therapeutic procedure. Complications occur rarely and they include bleeding, perforation and post-polypectomysyndrome. Incidence of perforation varies and is higher after therapeutic procedures such as polypectomy or endoscopic mucosal resection. The sigmoid colon is reported to be frequently involved [2].

Perforation may be caused by different mechanisms such as barotrauma, mechanical rupture, thermal injury during electrocautery and polypectomy. In our case, it is presumed that the perforation occurred during polypectomy. Thermal injury of the colonic wall during electrocoagulation for polypectomies is an important additional factor [3].

A second possibility is of an unnoticed perforation of a colonic diverticulum. Excessive pressure following insufflation may cause herniation of the mucosa of a false diverticulum making the mucosa permeable to the air without an evident point of perforation [4]. Alternatively mechanical rupture of a diverticulum is usuallythe result of excessive pressure against the colonic wall by the endoscope directly.

Perforations most commonly occur at points of previous weakness such as diverticula, previous colonic lesion sites, colitic areas, or neoplastic sites. Advanced age, comorbidities, and skills of the endoscopist further influence the risk of this complication [2].

Retroperitoneal perforations are unusual. Cirt et al. [3] reviewed literature from 1974 to 2006, 24 cases of retroperitoneal perforation have been reported. 14 of which were associated with polypectomies and only two were surgically managed.

How air enters and moves through the mediastinum can be understood by referring to the soft tissue compartments of the neck, thorax, and abdomen. The visceral space surrounding the trachea, oesophagus, and great vessels in the neck continues into the chest to envelop the mediastinal viscera and passes through the diaphragm with the oesophagus to communicate with the retroperitoneal space. Thus, it is possible for air that enters these tissue planes to track to any of the mediastinal structures.

Due to the rarity of this case, there is no ideal management.However, it is generally accepted that conservative management is perfectly reasonable in patients in good clinical condition in the absence of mechanical obstruction [3]. Success rate of non-operative management is approximately $33-73 \%$ [1]. Laparoscopic lavage has also been shown to be successful, leading to a good recovery without bowel resection. White et al. [5] showed the short term failures of laparoscopic washout were secondary to perforated cancer, fistula formation and inadequate washout leading to continued sepsis. Long-term problems may also exist with the technique. Resection and radiological drainage remain commonly used practices in the management of bowel perforation $[1,3]$.

With regards to our case, both intra- and extraperitoneal perforation was evident. Retroperitoneal involvement was evident in view of the subcutaneous emphysema. Intraperitoneal perforation was clinically suspected and confirmed with radiological investigation. No obvious perforation or contamination was noted on laparoscopy. Prompt recognition and management allowed us to minimise the consequences of the perforation and allowed a relatively quick recovery.

\section{Conclusion}

Perforations involving both the intra and extraperitoneal space are extremely rare. There are different management options available and the choice should be related to the clinical condition of the patient. Laparoscopicwashout and drain insertion is an emerging technique used in non-faeculent peritonitis. Evidence has shown it is a safe procedure, avoiding the need for a laparotomy and stoma formation.

\section{Consent}

Written informed consent was obtained from the patient for the publication of this report and accompanying images. 


\section{Conflict of interest}

We are not aware of any conflict of interest or any financial support received.

\section{References}

1. Lohsiriwat V (2010) Colonoscopic perforation: incidence, risk factors, management and outcome. See comment in PubMed Commons below World J Gastroenterol 16: 425-430.

2. Anderson ML1, Pasha TM, Leighton JA (2000) Endoscopic perforation of the colon: lessons from a 10-year study. See comment in PubMed Commons below Am J Gastroenterol 95: 3418-3422.
3. N Cirt, AS de Lajarte-Thirouard, D Olivié, M Pagenault, J.F Bretagne (2006) Subcutaneous emphysema, pneumomediastinum, pneumoperitoneum and retropneumoperitoneum following a colonoscopy with mucosectomy. GastroenterolClin Biol.30: 779-82.

4. HO Had Chih, S Burchell, P Morris, YU Mihae (1996) Colon perforation bilateral pneumothoraces, pneumopericardium, pneumomediastinum, and subcutaneous emphysema complicating endoscopic polypectomy: anatomic and management considerations. Am Surg 62: 770-4.

5. White SI, Frenkiel B, Martin PJ (2010) A ten-year audit of perforated sigmoid diverticulitis: highlighting the outcomes of laparoscopic lavage. See comment in PubMed Commons below Dis Colon Rectum 53: 1537-1541. 